\title{
OLAPARIB: A promising PARP Inhibitor for the treatment of ovarian cancer
}

\author{
Nikolaos Soupos, M.D. ${ }^{1 *}$, Konstantinos Laschos², Evangelos Bournakis ${ }^{3}$, \\ Euthymios Kostouros ${ }^{4}$, Gerasimos Aravantinos Dr., M.D. ${ }^{5}$ \\ 1 2nd Oncology Dpt, Cancer Hospital of Kifisia "Oi Agioi Anargiroi" \\ 22nd Oncology Dpt, Cancer Hospital of Kifisia "Oi Agioi Anargiroi" \\ ${ }^{3}$ Oncology Dpt. Areteio Hospital of Athens \\ ${ }^{4}$ Oncology Dpt., Department of Clinical Therapeutics at "Alexandra" Hospital, \\ Athens \\ ${ }^{5}$ Director, 2nd Oncology Dpt, Cancer Hospital of Kifisia "Oi Agioi Anargiroi"
}

Received 18 September 2015; Accepted 30 September 2015

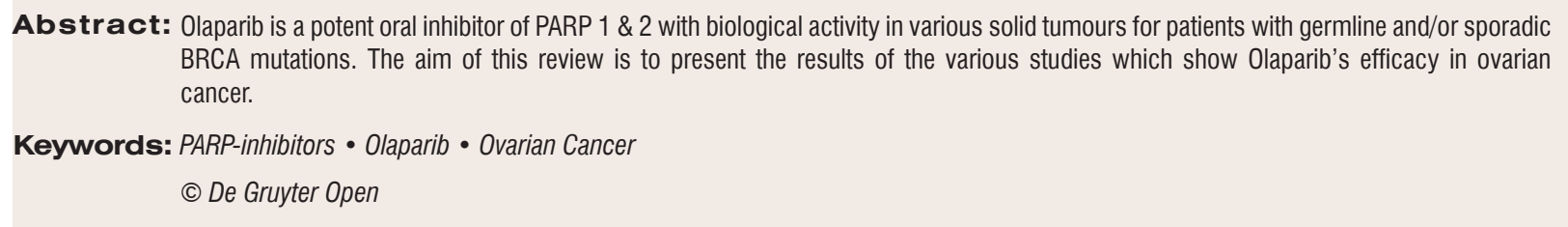

\section{Introduction}

Olaparib is a potent inhibitor of the enzyme poly-(ADPribose) polymerase 1 (PARP-1) and poly-(ADP-ribose) polymerase 2 (PARP-2), with biological activity in platinum-sensitive recurrent high-grade serous epithelial ovarian cancer (HGSOC), in patients with germline and/ or sporadic BRCA mutations and also in other solid tumours.

Ovarian cancer is the most common cause of death from gynaecologic tumours in the western world. It affects approximately 25,000 women annually in the United States and 800 women in Greece. At diagnosis, $75 \%$ are found to have advanced stage disease and thus require chemotherapy.

Although efforts have been made to improve the currently available chemotherapy drugs, their administration frequency and the use of other targeted (biological) drugs, ovarian cancer still remains the most lethal gynaecological malignancy with 15,000 deaths annually. The 'Standard of Care' in frontline chemotherapy after cytoreductive surgery remains systemic chemotherapy using the combination of a platinum and taxane-based regimen: more specifically, carboplatin and paclitaxel.

There are high response rates with first-line chemotherapy, which can reach up to $80 \%$; however, the majority of patients relapse and so undergo further lines of therapy, which results in progressively reduced progression-free survival (PFS), subjecting these patients to continued systemic therapy ultimately effecting their quality of life (QoL).

Maintenance therapy after first-line treatment has shown to prolong disease control. The need, however, to extend the time to progression (TTP) without extending the cytotoxic effect has given rise to a new group of targeted agents that are combined with the conventional platinum-based regimens. These targeted agents include angiogenesis inhibitors (tyrosine kinase inhibitors (TKIs) and anti-VEGFR (vascular endothelial growth factor)), 
PARP inhibitors and various others. Their activity is primarily focused on the maintenance period after firstline chemotherapy.

Women with germline mutations of BRCA 1, BRCA 2 or both have an increased risk of ovarian cancer compared to the general population particularly in those with invasive high-grade serous histology. In up to $50 \%$ of patients with HGSOC, the tumour cells are deficient in homologous recombination (HR) repair mechanism as a result of either germline or somatically acquired BRCA $1 / 2$ mutations, or epigenetic inactivation of BRCA 1 or other causes that can inactivate HR. The silencing mutations or dysfunction of the repair mechanism controlled by the BRCA 1/2-related pathways gives rise to the BRCAness phenotype. This phenotype profile appears to correlate with a higher response rate to platinumbased therapy and PARP inhibitors.

PARP inhibitors are a new class of anti-cancer agents that have been developed rapidly since 2005 based on the biological mechanism called synthetic lethality, known since 1944. Based on this, cell death occurs from a combination of mutations in two or more genes; however, a single mutation is unable to activate apoptosis.

PARP enzymes play an essential role in the repair of single-stranded DNA breaks (SSDB). DNA is unstable and alterations occur from three main causes: firstly, after exposure to environmental agents; secondly, as a result of by-products of normal cellular metabolism; and finally, by spontaneous disruption of chemical bonds in DNA. This causes a variety of lesions including base modifications, single-stranded breaks (SSB), double-stranded breaks and intrastrand or interstrand crosslinks. There are various repair mechanisms involved in repairing these lesions and maintaining genomic integrity. PARP 1 is crucial for the repair of SSBs especially by the process of base excision repair (BER). PARP 1 initiates the process by detecting and binding SSB. It's catalytic activity results in the polyADP ribosylation of PARP itself and other key proteins, enabling the repair of SSBs.

On the other hand, DSBs can be induced by X-rays or chemicals during repair of interstrand crosslink and during replication of SSBs converted into DSBs at replication forks and repaired by Homologous Recombination (HR). The role of BRCA 1 and BRCA 2 in DNA repair have been well documented, and they play an important role in the repair of DSBs by HR. Whilst normal cells can repair DSBs via the HR pathway (error free), loss of BRCA function forces cells to repair these DSBs via non-homologous end-joining or the single-strand annealing sub-pathway of HR, both the mechanisms are prone to error and genomic instability.
When exposed to PARP inhibitors, these cells are flooded with SSBs that are converted into DSBs at replication forks, and as a result, these BRCA-deficient cells are unable to use the HR pathway that would normally be the rescue mechanism. These changes lead to large numbers of chromatid aberrations and cause cell death. The use of PARP inhibitors in BRCA-deficient tumour cells is probably the first example of the clinical application of the concept of synthetic lethality.

Also, olaparib may act by working synergistically with chemotherapy whereby inhibition of mitosis leads to the inability of DNA repair or accumulation drives to apoptosis.

\section{Different Molecules - Common Targets}

Post the initial encouraging in vitro results, a new group of PARP enzyme inhibitors evolved and were studied in women with ovarian cancer.

Veliparib (ABT-888) showed positive results in Phase II trials of advanced or metastatic non-small cell lung cancer (especially in squamous cell histology) in combination with chemotherapy (studies M10-898 and NCT01386385), in breast cancer, predominantly triple negative (TNBC BRCA mutated) (Phase 1 and II: NCT01104259, NCT01149083, NCT01142379) and prostate cancer. Recently, a gynaecologic oncology group (GOG) Phase II trial showed positive results and in ovarian cancer (platinum sensitive and platinum resistant) in patients with BRCA mutations.

Niraparib (MK4827) has shown promising results in Phase II trials in patients with breast cancer (BRCA mutated) whilst a Phase III trial under EORTC is ongoing.

Rucaparib (CO338, AGO14699, PF1367388) based on the results of the interim analysis of the Phase II trial, ARIEL 2, received a breakthrough designation from the FDA in April 2015 as a treatment for women with BRCAmutated advanced ovarian cancer that had received at least two lines of platinum-based therapy. These results were presented at ASCO 2015. A Phase III trial, ARIEL 3 , is ongoing in order to validate the Phase II results. The recruitment is planned to conclude sometime in 2016.

AZD2461, a novel potent PARP inhibitor has a lower affinity for the transmembrane drug efflux transporter, P-glycoprotein (PGP), which is responsible for the clearance and subsequent resistance to PARP inhibitors. From pre-clinical data, it seems that it could be an active agent in the event of olaparib resistance. Currently, there is no available Phase I data.

CEP9722, a small molecule and prodrug of CEP8983 which is a PARP inhibitor, seems to show chemo- 
sensitized activity in various solid tumours, one of which is urothelial cancer.

Other molecules currently under investigation include E7449, E7016 and INO-1001.

Olaparib (AZD2281) has been extensively studied and has shown the most promising results from the Phase I and II trials in ovarian cancer.

\section{Chemical Compound Information}

Olaparib is an oral PARP inhibitor with a molecular weight of 434.7 Da and an IC ${ }_{50}$ for PARP-1 of $0.005 \mu \mathrm{M}$ and PARP-2 of $0.001 \mu \mathrm{M}$.

It was developed in experimental Granta-519 engrafted nonobese diabetic (NOD) or severe combined immunodeficiency (SCID) mice.

\section{Clinical Trials}

\section{Phase I Studies}

In a Phase I trial by Fong et al., ${ }^{1}$ the maximum tolerated dose (MTD) in 19 patients was defined at $400 \mathrm{mg}$ b.i.d. This dosage resulted in a $47 \%$ partial response (PR) in patients (9/19) with BRCA-mutated breast cancer, ovarian cancer and prostate cancer. Furthermore, 63\% (12/19) had derived a clinical benefit.

In another study of Fong et al., ${ }^{2}$ in 50 patients with recurrent BRCA-mutated ovarian cancer, unselected by platinum free interval, 39 of them received monotherapy with olaparib $200 \mathrm{mg}$ b.i.d and 11 received this dose after escalation. Forty percent of these patients showed either Response Evaluation Criteria In Solid Tumors (RECIST) or Ca125 response for a median duration of 28 weeks. Interestingly, this trial revealed an important association between platinum sensitivity and clinical benefit gained from olaparib. Specifically, the more platinum sensitive the disease, the higher the response rate to olaparib (23\% Response Rate in platinum refractory disease, $45 \%$ in platinum resistant and $69 \%$ in platinum sensitive).

\section{Phase II Studies}

\section{Non-randomised Studies}

Audeh and colleagues ${ }^{3}$ in a non-randomized study in patients with recurrent ovarian cancer with BRCA mutations showed a dose-response relationship with olaparib. Patients were randomized into two groups : the first group received $400 \mathrm{mg}$ b.i.d and the second group received $100 \mathrm{mg}$ b.i.d. The patients in the first group showed RR of $33 \%$ in comparison to $13 \%$ for the second group. Furthermore, the patients who received $100 \mathrm{mg}$ b.i.d showed a higher rate of progression in 16 weeks (65\% vs $33 \%)$.

However, the results of this study need to be interpreted cautiously because, firstly, it was a nonrandomized study and, secondly, at the initial study design, the group that received $100 \mathrm{mg}$ b.i.d included patients with poorer prognostic features. As well, it should be noted that the RRs in both platinum-sensitive and platinum-resistant patients were similar (38\% vs $30 \%$, respectively) which is in contrast to the results that were observed in other Phase I studies.

In another Phase II study by Gelmon et al., ${ }^{4}$ 64 patients with relapsed ovarian cancer independent of BRCA mutations and independent of platinum sensitivity received olaparib $400 \mathrm{mg}$ b.i.d. The results interestingly showed an efficacy to olaparib even in wild-type BRCA (wtBRCA) patients.

Specifically, the RRs of imaging (based on RECIST) were $41 \%$ and $24 \%$ in mutated BRCA and wtBRCA, respectively.

It should be noted that in the meta-analysis of the wtBRCA population, the subgroup with platinumsensitive disease demonstrated RRs of $50 \%$ vs $4 \%$ in patients with platinum resistance.

At the beginning of 2015, Kaufman et al., ${ }^{5}$ published an interesting Phase II study that showed the efficacy of olaparib in a variety of solid tumours carrying germline BRCA mutations. More specifically, that multicenter study included 298 patients with BRCA 1/2 mutations. Of these, 193 patients were platinum resistant, 62 were heavily pretreated ( $\geq 3$ lines chemotherapy) breast cancer, 23 patients with pancreatic cancer relapsed after gemcitabine-based therapy, 6 patients with prostate cancer (CRPC and relapsed after first-line chemotherapy) and another 12 patients with other solid tumours. All the patients received olaparib $400 \mathrm{mg}$ b.i.d and the primary end point was the RR. The total RR results were $26.2 \%(78 / 298 ; 95 \% \mathrm{Cl}, 21.3$ to 31.6$)$, whilst the results for each of the specific solid tumour groups were $31.1 \%$ (60 /193; 95\% Cl, 24.6 to 38.1$), 12.9 \%$ (8 / $62 ; 95 \% \mathrm{Cl}, 5.7$ to 23.9$), 21.7 \%$ (5/23; $95 \% \mathrm{Cl}, 7.5$ to $43.7)$ and $50.0 \%(4 / 8 ; 95 \% \mathrm{Cl}, 15.7$ to 84.3$)$ in ovarian, breast, pancreatic and prostate cancers, respectively.

\section{Randomised Studies}

Several randomized Phase II studies have already been published whilst others are ongoing in order to evaluate the efficacy of olaparib in recurrent ovarian cancer.

A randomized Phase II study was published by Kaye et al. in $2008,{ }^{6}$ which recruited 97 patients with recurrent ovarian cancer that relapsed in $\leq 12$ months 
from the last platinum-based chemotherapy carrying BRCA 1 and BRCA 2 mutations who had not previously been treated with pegylated liposomal doxorubicin (PLD). The randomization was designed with three arms: Arm A, 32 patients received $200 \mathrm{mg}$ olaparib b.i.d; Arm B, 32 patients received $400 \mathrm{mg}$ b.i.d olaparib; Arm C, 33 patients received $50 \mathrm{mg} / \mathrm{m}^{2}$ every 28 days. The results showed a PFS of $6.5,8.8$ and 7.1 months for olaparib $200 \mathrm{mg}, 400 \mathrm{mg}$ and PLD, respectively, with ORR $25 \%, 31 \%$ and $18 \%$, respectively. Furthermore, a smaller percentage of patients $28.1 \%$ with $200 \mathrm{mg}$ and $34.4 \%$ with $400 \mathrm{mg}$ in comparison $45.5 \%$ PLD presented new lesions.

Even though the primary end point of the study was not met, it is worth noting that firstly, the efficacy of olaparib in BRCA-mutated ovarian cancer is comparable to PLD. Secondly, confirmation of the ORR of olaparib at the $400 \mathrm{mg}$ b.i.d dose (31\%) was similar to that described in previous studies. Finally and more importantly, looking ahead at future studies, anthracyclines (specifically PLD) are becoming increasingly important in BRCAmutated ovarian cancer with the combination of olaparib and PLD creating for interesting further research.

Another double-blinded Phase II study from Ledermann et al. ${ }^{7}$ between 2008 and 2010 included 265 patients unselected for BRCA status, 136 received $400 \mathrm{mg}$ b.i.d olaparib and 129 received placebo (1:1) as maintenance therapy. The patients had platinumsensitive serous ovarian cancer and had received two or more lines of platinum-based chemotherapy and had at least reached a PR to the most recent platinumbased regimen. The initial interim analysis of the study population showed a statistically significant increased PFS for the arm with olaparib versus placebo (8.4 vs 4.8 months, [HR]: 0.35 ; $95 \% \mathrm{Cl}: 0.25-0.49 ; p<0.00001)$

After the identification of the BRCA status of the study population and the subgroup analysis, it appeared that patients with BRCA mutations had a median PFS even longer in the olaparib arm (11.2 vs 4.3 months HR: 0.35 , $\mathrm{p}<0.00001)$. Also in the wtBRCA subgroup, a PFS was longer in the olaparib arm (7.4 vs 5.5 months, $p<0.0075)$. Even though the above results were very encouraging, they did not, however, translate into an OS benefit (in $58 \%$ maturity). At this point, the authors emphasized a bias of the study was that $23 \%(14 / 62)$ of the patients with BRCA mutations who were included in the placebo arm, after showing progression, were crossed over to receive olaparib and this ultimately may have influenced the OS benefit. A take home message of this study is that in patients with platinum-sensitive relapsed serous ovarian cancer, a personalized therapeutic approach, based on BRCA mutation status, is advised to maximise the clinical benefit.
In another randomized Phase II study from Oza et al. ${ }^{8}$ of the initial 173 patients, 162 were selected with recurrent high-grade platinum-sensitive serous ovarian cancer that had received up to three lines of platinum-based therapy. All the patients were disease free in the last 6 months prior to their recruitment. The randomization was 1:1. Eighty-one patients received six courses of paclitaxel $\left(175 \mathrm{mg} / \mathrm{m}^{2}\right)$ and carboplatin (4 AUC) combined with $200 \mathrm{mg}$ olaparib b.i.d and 81 patients received six courses of paclitaxel $\left(175 \mathrm{mg} / \mathrm{m}^{2}\right)$ combined with carboplatin (6 AUC). The patient selection was independent or unknown of BRCA status and the primary end point of the study was PFS.

The results showed added benefit for the olaparib plus chemotherapy arm versus chemotherapy alone (12.2 vs 9.6 months, $p<0.0012$ ), even though the carboplatin dosage was lower. The benefit was more evident in the population with BRCA mutations where the olaparib arm showed superiority at the event-free survival over 12 months of $70 \%$ vs $12.5 \%$ of chemotherapy alone. It is obvious from this study that the benefit of adding olaparib to chemotherapy and particularly during the maintenance phase offered a better DCR in the population who had BRCA mutations. Regarding the toxicity profile, the concomitant administration of olaparib with conventional chemotherapy seems to be well tolerated with more frequent Grade 3 or higher adverse events: neutropenia $(43 \%$ vs $35 \%$ of chemotherapy only) and anaemia ( $9 \%$ vs $7 \%$ ). Based on these results, future trial design with PARP inhibitors administered after chemotherapy as maintenance seems feasible.

Another Phase II study by Liu et al. ${ }^{9}$ looked at the combination of olaparib and cediranib. Cediranib is an oral tyrosine kinase inhibitor directed against the VEGF receptor 1,2 and 3 . There is data available in recurrent ovarian cancer with cediranib as monotherapy with RRs as high as $17 \%$ with a median PFS 5.2 months.

From available preclinical data and Phase I studies in triple negative breast cancer and recurrent ovarian cancer, it appeared that the combination of olaparib with cediranib showed RRs of $44 \%$. In the Liu study, patients who were included had relapsed platinum-sensitive high-grade serous ovarian cancer and endometrioid histology. The selection was not based on BRCA status. From the study design, the patients were randomised to receive either olaparib $400 \mathrm{mg}$ b.i.d or olaparib $200 \mathrm{mg}$ b.i.d in combination with cediranib $30 \mathrm{mg}$ daily. The RRs for the general study population who received combination therapy versus monotherapy was $79.6 \%$ versus $47.8 \%(p=0.002)$ and the PFS was 17.7 versus 9 months, respectively.

An interesting point to note from this study is that the post-hoc analysis result showed a superior efficacy in the 
wtBRCA subgroup where the PFS for the combination was up to 16.5 versus 5.7 months for the monotherapy group $(p=0.008)$. Even though the differences are not statistically significant, they are still worth noting for the mBRCA population (19.4 vs 16.5 months in favour of the combination therapy; $p=0.16)$.

\section{Phase III Studies}

Before the encouraging Phase II study results, various phase III studies are ongoing in order to confirm the available data and to add further evidence regarding the use of olaparib as a maintenance therapy.

The SOLO-1 (GOG3004) study is a randomized double-blinded 2:1 study including patients with newly diagnosed high-grade serous and endometrioid ovarian or fallopian tubes, stage III-IV cancer with BRCA mutations. In this study, patients with at least a PR at the completion of frontline chemotherapy randomised (2:1) to receive olaparib $150 \mathrm{mg}$ b.i.d or placebo until disease progression. The primary end point of the study is PFS.

Similar is the studies of olaparib in ovarian cancer 2 (SOLO-2) study trial design, with the difference being that patients included have relapsed platinum-sensitive disease and have received at least two platinum-based regimens.

\section{Toxicity and Tolerability}

From the PARP inhibitors, the most frequent adverse events include myelotoxicity and gastrointestinal disorders. Of special interest is myelotoxicity, particularly in patients with overlapping toxicity from conventional chemotherapy or heavy pretreatment whereby their bone marrow reserves are exhausted.

\section{References}

[1] Fong PC, Yap TA, Boss DS, Carden CP, MerguiRoelvink M, Gourley C, De Greve J, Lubinski J, Shanley S, Messiou C, A'Hern R, Tutt A, Ashworth A, Stone J, Carmichael J, Schellens JH, de Bono JS, Kaye SB. Poly(ADP)-ribose polymerase inhibition: frequent durable responses in BRCA carrier ovarian cancer correlating with platinum-free interval. J Clin Oncol. 2010 May 20;28(15):2512-9. doi: 10.1200/ JCO.2009.26.9589. Epub 2010 Apr 20.

[2] Fong PC, Yap TA, Boss DS, Carden CP, MerguiRoelvink M, Gourley C, De Greve J, Lubinski J, Shanley S, Messiou C, A'Hern R, Tutt A, Ashworth A, Stone J, Carmichael J, Schellens JH, de Bono JS, Kaye SB. Poly(ADP)-ribose polymerase inhibition:
Regarding the gastrointestinal toxicity, taking into consideration the nature of the disease and the existing disorders because of the extended peritoneal disease could be a limiting factor for the use of these agents. This was also observed in the Ledermann et at. ${ }^{10}$ study where the discontinuation rates were more frequent in the olaparib arm versus placebo $(27.9 \%$ vs $8.6 \%)$ and dose reductions, respectively, were $22.8 \%$ versus $4.7 \%$.

The more common causes of discontinuation as reported in this study were vomiting, nausea and fatigue, with grade 3-4 toxicity $35.3 \%$ for the olaparib arm versus $20.3 \%$ for placebo.

Haematological toxicities in heavily pretreated patients as reported in the Kaufman et al. study where anaemia of grade 3 or more was observed in $18.7 \%$, fatigue $60 \%$, nausea $61.7 \%$ and vomiting $38.9 \%$.

\section{Conclusions}

PARP in patients with somatic or germline mutations of BRCA is an attractive therapeutic target showing encouraging results derived from studies looking at various solid tumours.

This field includes a continued increasing number of evolving new agents whereby in the future, their target will be to overcome the resistance to PARP inhibitors.

Olaparib is the first agent supported by many studies particularly in ovarian cancer. It has an acceptable toxicity profile and its oral formulation renders it a preferred option for maintenance therapy. Evidence from the phase II studies supports that it is a therapy most effective in patients with BRCA mutations.

frequent durable responses in BRCA carrier ovarian cancer correlating with platinum-free interval. J Clin Oncol. 2010 May 20;28(15):2512-9. doi: 10.1200/ JCO.2009.26.9589. Epub 2010 Apr 20.

[3] Audeh MW, Carmichael J, Penson RT, Friedlander M, Powell B, Bell-McGuinn KM, Scott C, Weitzel JN, Oaknin A, Loman N, Lu K, Schmutzler RK, Matulonis $U$, Wickens $M$, Tutt A. Oral poly(ADPribose) polymerase inhibitor olaparib in patients with BRCA1 or BRCA2 mutations and recurrent ovarian cancer: a proof-of-concept trial. Lancet. 2010 Jul 24;376(9737):245-51. doi: 10.1016/ S0140-6736(10)60893-8. Epub 2010 Jul 6. 
[4] Gelmon KA, Tischkowitz M, Mackay H, Swenerton K, Robidoux A, Tonkin K, Hirte H, Huntsman D, Clemons M, Gilks B, Yerushalmi R, Macpherson E, Carmichael J, Oza A. Olaparib in patients with recurrent high-grade serous or poorly differentiated ovarian carcinoma or triple-negative breast cancer: a phase 2 , multicentre, open-label, non-randomised study. Lancet Oncol. 2011 Sep;12(9):852-61. doi: 10.1016/S1470-2045(11)70214-5. Epub 2011 Aug 19.

[5] Kaufman B1, Shapira-Frommer R1, Schmutzler RK1, Audeh MW1, Friedlander M1, Balmaña J1, Mitchell G1, Fried G1, Stemmer SM1, Hubert A1, Rosengarten O1, Steiner M1, Loman N1, Bowen K1, Fielding A1, Domchek SM2. Olaparib monotherapy in patients with advanced cancer and a germline BRCA1/2 mutation. J Clin Oncol. 2015 Jan 20;33(3):244-50. doi: 10.1200/JCO.2014.56.2728. Epub 2014 Nov 3.

[6] Kaye SB, Lubinski J, Matulonis U, Ang JE, Gourley C, Karlan BY, Amnon A, Bell-McGuinn KM, Chen LM, Friedlander M, Safra T, Vergote I, Wickens M, Lowe ES, Carmichael J, Kaufman B. Phase II, open-label, randomized, multicenter study comparing the efficacy and safety of olaparib, a poly (ADP-ribose) polymerase inhibitor, and pegylated liposomal doxorubicin in patients with BRCA1 or BRCA2 mutations and recurrent ovarian cancer. J Clin Oncol. 2012 Feb 1;30(4):372-9. doi: 10.1200/ JCO.2011.36.9215. Epub 2011 Dec 27.

[7] Ledermann J1, Harter P, Gourley C, Friedlander M, Vergote I, Rustin G, Scott C, Meier W, ShapiraFrommer R, Safra T, Matei D, Macpherson E, Watkins $\mathrm{C}$, Carmichael J, Matulonis U. Olaparib maintenance therapy in platinum-sensitive relapsed ovarian cancer. N Engl J Med. 2012 Apr 12;366(15):138292. doi: 10.1056/NEJMoa1105535. Epub $2012 \mathrm{Mar}$ 27.

[8] Oza AM, Cibula D, Benzaquen AO, Poole C, Mathijssen RH, Sonke GS, Colombo N, Špaček J, Vuylsteke P, Hirte H, Mahner S, Plante M, Schmalfeldt B, Mackay H, Rowbottom J, Lowe ES, Dougherty B, Barrett JC, Friedlander M. Olaparib combined with chemotherapy for recurrent platinum-sensitive ovarian cancer: a randomised phase 2 trial. Lancet Oncol. 2015 Jan;16(1):87-97. doi: 10.1016/S1470-2045(14)71135-0. Epub 2014 Dec 4.

[9] Ledermann J1, Harter P2, Gourley C3, Friedlander M4, Vergote 15, Rustin G6, Scott CL7, Meier W8, Shapira-Frommer R9, Safra T10, Matei D11, Fielding A12, Spencer S12, Dougherty B13, Orr M12, Hodgson D12, Barrett JC13, Matulonis U14.
Olaparib maintenance therapy in patients with platinum-sensitive relapsed serous ovarian cancer: a preplanned retrospective analysis of outcomes by BRCA status in a randomised phase 2 trial. Lancet Oncol. 2014 Jul;15(8):852-61. doi: 10.1016/S14702045(14)70228-1. Epub 2014 May 31.

[10] Ledermann J1, Harter P2, Gourley C3, Friedlander M4, Vergote 15, Rustin G6, Scott CL7, Meier W8, Shapira-Frommer R9, Safra T10, Matei D11, Fielding A12, Spencer S12, Dougherty B13, Orr M12, Hodgson D12, Barrett JC13, Matulonis U14. Olaparib maintenance therapy in patients with platinum-sensitive relapsed serous ovarian cancer: a preplanned retrospective analysis of outcomes by BRCA status in a randomised phase 2 trial. Lancet Oncol. 2014 Jul;15(8):852-61. doi: 10.1016/S14702045(14)70228-1. Epub 2014 May 31.

[11] Jemal A1, Bray F, Center MM, Ferlay J, Ward E, Forman D. Global cancer statistics.CA Cancer J Clin. 2011 Mar-Apr;61(2):69-90. doi: 10.3322/ caac.20107. Epub2011 Feb 4.

[12] Fung-Kee-Fung M, Oliver T, Elit L, Oza A, Hirte HW, Bryson P. Optimal chemotherapy treatment for women with recurrent ovarian cancer. Curr Oncol. 2007 Oct;14(5):195-208.

[13] Parmar MK, Ledermann JA, Colombo N, du Bois A, Delaloye JF, Kristensen GB, Wheeler S, Swart AM, Qian W, Torri V, Floriani I, Jayson G, Lamont A, Tropé C. Paclitaxel plus platinum-based chemotherapy versus conventional platinum-based chemotherapy in women with relapsed ovarian cancer: the ICON4/ AGO-OVAR-2.2 trial.; ICON and AGO Collaborators. Lancet. 2003 Jun 21;361(9375):2099-106.

[14] Moynahan ME1, Chiu JW, Koller BH, Jasin M.Brca1 controls homology-directed DNA repair. Mol Cell. 1999 Oct;4(4):511-8.

[15] Moynahan ME, PierceAJ, Jasin M. BRCA2 is required for homology-directed repair of chromosomal breaks. Mol Cell. 2001 Feb;7(2):263-72.

[16] Konstantinopoulos PA1, Spentzos D, Karlan BY, Taniguchi T, Fountzilas E, Francoeur N, Levine DA, Cannistra SA. Gene expression profile of BRCAness that correlates with responsiveness to chemotherapy and with outcome in patients with epithelial ovarian cancer. J Clin Oncol. 2010 Aug 1;28(22):3555-61. doi: 10.1200/JCO.2009.27.5719. Epub 2010 Jun 14.

[17] Liu JF, Tolaney SM, Birrer M, Fleming GF, Buss MK, Dahlberg SE, Lee H, Whalen C, Tyburski K, Winer E, Ivy P, Matulonis UA.A Phase 1 trial of the poly(ADP-ribose) polymerase inhibitor olaparib (AZD2281) in combination with the anti-angiogenic cediranib (AZD2171) in recurrent epithelial 
ovarian or triple-negative breast cancer. Eur J Cancer. 2013 Sep;49(14):2972-8. doi: 10.1016/j. ejca.2013.05.020. Epub 2013 Jun 27.

[18] Zhang S, Royer R, Li S, McLaughlin JR, Rosen B, Risch HA, Fan I, Bradley L, Shaw PA, Narod SA. Frequencies of BRCA1 and BRCA2 mutations among 1,342 unselected patients with invasive ovarian cancer. Gynecol Oncol. 2011 May 1;121(2):353-7. doi: 10.1016/j.ygyno.2011.01.020. Epub 2011 Feb 15.

[19] Camille C Gunderson, Kathleen N Moore Olaparib: an oral PARP-1 and PARP-2 inhibitor with promising activity in ovarian cancer. Future Oncology, Vol. 11, No. 5, Pages 747-757.
[20] Ang JE, Gourley C, Powell CB, High H, ShapiraFrommer R, Castonguay V, De Greve J, Atkinson T, Yap TA, Sandhu S, Banerjee S, Chen LM, Friedlander ML, Kaufman B, Oza AM, Matulonis U, Barber LJ, Kozarewa I, Fenwick K, Assiotis I, Campbell J, Chen L, de Bono JS, Gore ME, Lord CJ, Ashworth A, Kaye SB. Efficacy of chemotherapy in BRCA1/2 mutation carrier ovarian cancer in the setting of PARP inhibitor resistance: a multiinstitutional study. Clin Cancer Res. 2013 Oct 1;19(19):5485-93. doi: 10.1158/1078-0432.CCR13-1262. Epub 2013 Aug 6. 